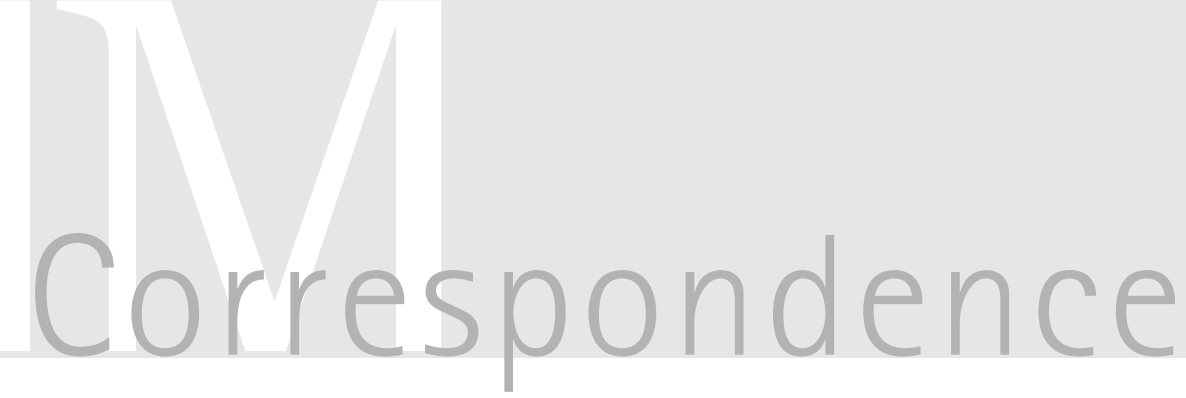

\title{
Wet mounting using iodine-glycerol provides a semi-permanent preparation for microscopic observation of faecal parasites
}

Parasites of diarrhoeal aetiology are widespread in water supplies, infecting a significant proportion of the human population in third-world countries (Vignesh et al., 2007), especially across the Asian subcontinent. With an everincreasing population leading to overcrowding and unhygienic practices, these parasites pose a serious threat that is compounded by limited resources. Competency in the diagnosis and proficiency of laboratories in such peripheral settings remain questionable and may be attributed to these limited resources. Simple and cost-effective diagnostic methods may provide a solution to these difficulties.

Microscopic observation of wet mounts remains the most widely used method for identifying ova and cysts of parasites from stool specimens (Valentina \& Lalitha, 2001). The classical technique described for the microscopic examination of parasites is the iodine mount method, which facilitates the differentiation and identification of parasites by characteristic morphological features and details of internal structures. The method is easy, less time-consuming and inexpensive, allowing direct visualization of parasitic ova and cyst morphology. The only disadvantage to this technique is that the preparation dries within a few minutes, rendering it unreadable. Making a fresh preparation is required when the slide is to be viewed later for consultation or demonstration purposes. Remaking the preparation consumes considerable resources and technician's time. We propose a very simple, inexpensive alternative to the iodine mount that lasts

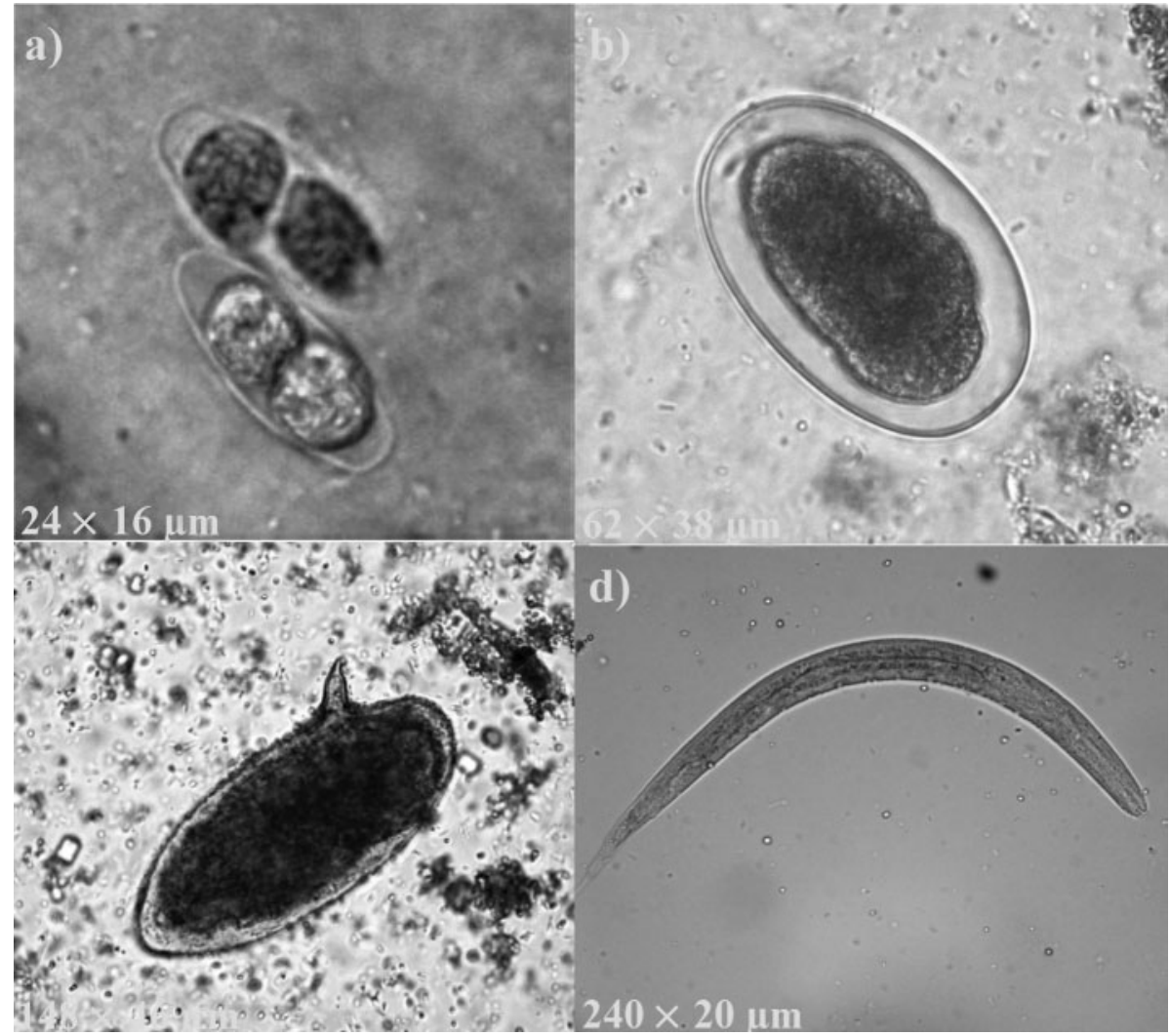

Fig. 1. Semi-permanent wet mount preparation using iodine-glycerol solution to detect oocysts of Isospora belli (a), ova of hookworm (b) and Schistosoma mansoni (c) and rhabditiform larvae of Strongyloides stercoralis (d). The figure shows efficiently differentiated internal structures of the parasites under the high-power objective of a binocular microscope (Leica DMLS). The prepared wet mounts lasted without drying despite storage for 2 days at $25 \pm 2{ }^{\circ} \mathrm{C}$, while the iodine wet mounts dried up in $<10 \mathrm{~min}$ at the above temperature (not shown). 
longer without drying, which will facilitate any subsequent expert confirmation. Various studies have documented the advantages of using lactophenol cotton blue (LPCB) for preparing wet mounts for parasitic identification (Parija \& Prabhakar, 1995; Parija et al., 2003). Wet mount preparations made using LPCB do not dry up due to the presence of glycerol in it. Glycerol, by its hygroscopic nature, absorbs water molecules from the environment and prevents drying out of wet mounts. Considering the cost, difficulty in availability and hazardous nature of the ingredients of LPCB, we prepared a mounting solution of $0.25 \%$ glycerol (Himedia) in iodine solution (Himedia) used conventionally in peripheral laboratories. Wet mounts prepared with the iodine-glycerol solution were compared with conventional iodine mounts and were evaluated for any visible differences in the clarity of mount and the visual details of morphology and internal structures of parasites under low- and high-power microscopy (Leica DMLS). Using these preparations, we examined the time required to visualize certain morphological forms of representative parasites, viz. oocysts of Isospora belli, ova of hookworm and Schistosoma mansoni and rhabditiform larvae of Strongyloides stercoralis, and the time required for the preparations to dry out at ambient tropical laboratory temperature $\left(25 \pm 2{ }^{\circ} \mathrm{C}\right)$. We found that the iodine-glycerol mount differentiated the internal structures of the parasites clearly and facilitated accurate detection and identification (Fig. 1) as efficiently as the conventional iodine mount. The iodine-glycerol solution lasted longer without drying out, even after 2 days, while the iodine wet mounts dried up in $<10 \mathrm{~min}$.

Parasites have a series of distinct developmental stages in their life cycle. Based on their stage of development, unique life cycle and time delay between stool specimen collection and observation, parasites may be observed in different stages and morphology, namely ova, cysts, larvae and trophozoites. Under-diagnosis of these diverse morphological forms is reportedly common in peripheral settings, where difficulties related to laboratory resources and patient follow-up are believed to be the basic shortcomings on the way to prompt and accurate diagnosis. Furthermore, basic diagnostic tests in these settings might often require expert opinion before declaration of results. Therefore, the proposed simple, easily available, mounting medium that offers considerable longevity could be useful for enabling cost-effective diagnosis of common faecal parasites.

\section{Ramachandran Vignesh, Ramalingam Sekar,}

Esaki Muthu Shankar, Nagalingeswaran Kumarasamy, Kailapuri G. Murugavel, Paulas Irene, Suniti Solomon and Pachamuthu Balakrishnan

YRG Centre for AIDS Research and Education, VHS Campus, Rajiv Gandhi Salai, Taramani, Chennai 600 113, India

Correspondence:

Pachamuthu Balakrishnan

(bala@yrgcare.org)

Parija, S. C. \& Prabhakar, P. K. (1995).

Evaluation of lacto-phenol cotton blue for wet mount preparation of feces. J Clin Microbiol 33, 1019-1021.

Parija, S. C., Shivaprakash, M. R. \& Jayakeerthi, S. R. (2003). Evaluation of lacto-phenol cotton blue (LPCB) for detection of Cryptosporidium, Cyclospora and Isospora in the wet mount preparation of stool. Acta Trop 85, 349-354.

Valentina, G. \& Lalitha, M. K. (2001).

Parasitology. In Myer's and Koshi's Manual of Diagnostic Procedures in Medical Microbiology and Immunology/Serology, pp. 31-37. Edited by E. Mathai. Vellore, India: Christian Medical College and Hospital.

Vignesh, R., Balakrishnan, P., Shankar, E. M., Murugavel, K. G., Hanas, S., Cecelia, A. J., Thyagarajan, S. P., Solomon, S. \& Kumarasamy, N. (2007). High proportion of isosporiasis among HIV-infected patients with diarrhea in Southern India. Am J Trop Med Hyg 77, 823-824. 